\title{
Coherent trapping of atomic populations
}

\author{
H. R. Gray, R. M. Whitley, ${ }^{*}$ and C. R. Stroud, Jr. \\ The Institute of Optics, University of Rochester, Rochester, New York 14627
}

Received August 31, 1978

\begin{abstract}
It is often desirable in laser spectroscopy and isotope separation to extract as much as possible of an atomic or molecular population that is distributed among a number of ground-state sublevels and low-lying metastable levels. We describe a form of coherent trapping that occurs when multiple resonant laser beams are used to couple the various ground states to a common upper level. This effect prevents the extraction of the entire population. We then study the effect with two dye lasers and an atomic beam and suggest possible ways to maximize the pumping efficiency.
\end{abstract}

When two cw lasers are tuned so that they couple two different ground-state sublevels to a common upper level, there is no steady-state population in the upper level. (See Fig. 1 for the energy-level configuration.) This effect, which holds even for intense laser fields, might be termed coherent trapping of atomic populations. It is due to optical pumping of the ground-state sublevels into a coherent superposition state that is decoupled from the laser fields. The theory of the effect has been worked out by Arimondo and Orriols ${ }^{1}$ and by Whitley. ${ }^{2}$ The source of the effect is easily seen by writing the wavefunction in the interaction picture,

$\psi(r, t)=a_{1}(t) \exp \left(-i \omega_{1} t\right) \psi_{1}(r)$

$+a_{2}(t) \exp \left(-i \omega_{2} t\right) \psi_{2}(r)+a_{3}(t) \exp \left(-i \omega_{3} t\right) \psi_{3}(r)$,

and substituting it into Schrödinger's equation. Here we have taken the atomic-energy eigenfunctions as $\psi_{i}(r)$ and the energy of the various states as $E_{i}=\hbar \omega_{i}, i=$ $1,2,3$. The amplitudes then satisfy the equations of motion

$$
\begin{aligned}
\dot{a}_{1}= & \left(i \mu_{1} \epsilon_{a} / \hbar\right) \exp \left(i \delta_{a} t\right) a_{2}, \\
\dot{a}_{2}= & \left(i \mu_{1} \epsilon_{a} / \hbar\right) \exp \left(-i \delta_{a} t\right) a_{1} \\
& \quad+\left(i \mu_{2} \epsilon_{b} / \hbar\right) \exp \left(-i \delta_{b} t\right) a_{3}, \\
\dot{a}_{3}= & \left(i \mu_{2} \epsilon_{b} / \hbar\right) \exp \left(i \delta_{b} t\right) a_{2},
\end{aligned}
$$

where we have taken the applied field to be

$$
E(t)=\epsilon_{a} \exp \left(-i \omega_{a} t\right)+\epsilon_{b} \exp \left(-i \omega_{b} t\right)+\text { c.c. }
$$

and we have neglected the counterrotating terms. The two dipole matrix elements, which are assumed real for convenience, are denoted by $\mu_{i}, i=1,2$, while the detuning of the two lasers from resonance, $\delta_{a}$ and $\delta_{b}$, are defined in Fig 1.

Examination of Eqs. (2a) and (2c) shows that there is a constant of the motion when $\delta_{a}=\delta_{b} \equiv \delta$, i.e., when the lasers are tuned to the two-photon resonance. The effect of the constant is most easily seen if we introduce two new amplitudes,

$$
\begin{aligned}
& r(t)=a_{1}(t) \cos \theta-a_{3}(t) \sin \theta, \\
& s(t)=a_{1}(t) \sin \theta+a_{3}(t) \cos \theta,
\end{aligned}
$$

where the angle $\theta$ is defined by

$$
\tan \theta=\left(\mu_{1} \epsilon_{a} / \mu_{2} \epsilon_{\mathrm{b}}\right) \text {. }
$$

The equations of motion for the amplitudes of the two ground states are then expressible in terms of $r(t)$ and $s(t)$ :

$$
\begin{aligned}
& \dot{r}(t)=0, \\
& \dot{s}(t)=i R \exp (i \delta t) a_{2}(t),
\end{aligned}
$$

where $R$ is the generalized Rabi frequency

$$
R=(1 / \hbar)\left[\left(\mu_{1} \epsilon_{a}\right)^{2}+\left(\mu_{2} \epsilon_{b}\right)^{2}\right]^{1 / 2} .
$$

One linear combination of the ground states is coupled to the excited state by the applied fields, and the other linear combination is decoupled entirely. The population initially in the linear combination,

$$
r(0)=a_{1}(0) \cos \theta-a_{3}(0) \sin \theta,
$$

remains there. If the initial states are not prepared coherently, this means that half of the population remains in the ground state and nothing is gained by the application of two lasers rather than one.

We have not included spontaneous or collisional damping in the calculations, but these are included in the calculations of Refs. 1 and 2. The result of including damping is that the population initially in the linear combination of states $s$ is optically pumped in a few lifetimes into the decoupled linear combination $r$. In the steady state there is no population in level 2 or in the linear combination $s$. All of it is in the linear combination $r$. The atom is decoupled from the field with all the population in a particular coherent superposition of the ground states. The asymptotic steady-state wavefunction is

$$
\begin{aligned}
\lim _{t \rightarrow \infty} \psi(r, t)=\cos \theta \exp \left(-i \omega_{1} t\right) \psi_{1}(r) & \\
& -\sin \theta \exp \left(-i \omega_{3} t\right) \psi_{3}(r) .
\end{aligned}
$$

If the fields are detuned from the two-photon resonance, the constant of the motion is not exact and there is some steady-state population in the excited state. Efficient extraction of the atomic population from the ground state into the excited state and perhaps from 


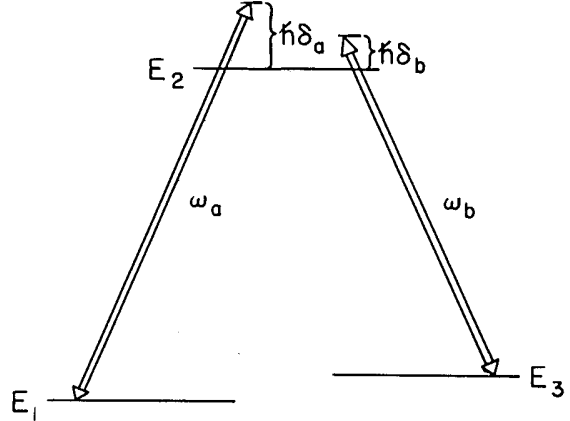

Fig. 1. Energy-level configuration. $E_{1}$ and $E_{3}$ are nondegenerate sublevels of the ground state. $E_{2}$ is a common excited state. Two monochromatic lasers of frequency $\omega_{a}$ and $\omega_{b}$ are tuned $\delta_{a}$ and $\delta_{b}$ off resonance.

there to some higher bound or continuum level is accomplished by detuning the lasers from exact resonance. Whitley ${ }^{2}$ has derived the steady-state solutions for arbitrary detunings and radiative damping. The population in level 2 is given by

$$
\begin{gathered}
\sigma_{22}=N_{22} / D \\
N_{22}=4 \alpha^{2} \beta^{2}\left(\Gamma_{a}+\Gamma_{b}\right)\left(\delta_{a}-\delta_{b}\right)^{2}, \\
D=\left(\delta_{a}-\delta_{b}\right)^{2}\left\{8 \alpha^{2} \beta^{2}\left(\Gamma_{a}+\Gamma_{b}\right)+16 \alpha^{2} \Gamma_{b}\left[\left(\Gamma_{a}+\Gamma_{b}\right)^{2}\right.\right. \\
\left.\left.+\delta_{b}{ }^{2}\right]+16 \beta^{2} \Gamma_{a}\left[\left(\Gamma_{a}+\Gamma_{b}\right)^{2}+\delta_{a}{ }^{2}\right]\right\} \\
+8\left(\delta_{a}-\delta_{b}\right)\left(\alpha^{4} \delta_{b} \Gamma_{b}-\beta^{4} \delta_{a} \Gamma_{a}\right) \\
+\left(\alpha^{2} \Gamma_{b}+\beta^{2} \Gamma_{a}\right)\left(\alpha^{2}+\beta^{2}\right)^{2}
\end{gathered}
$$

where $\alpha$ and $\beta$ are the Rabi frequencies of the fields at frequencies $\omega_{a}$ and $\omega_{b}$, respectively. The $\Gamma$ 's are half of the corresponding Einstein $A$ coefficients.

We have tested these predictions by tuning two $\mathrm{cw}$ dye lasers, frequency stabilized to $\pm 1 \mathrm{MHz}$, to two hyperfine components of the sodium $D_{1}$ line. The transitions from the $F=1$ and $F=2$ ground states to the $F$ $=2$ component of the $3 P_{1 / 2}$ level were used. Two linearly polarized laser beams were brought in together to illuminate an atomic beam (collimated to $0.8 \mathrm{mrad}$ ) at right angles to the atomic velocity vectors. The fluorescence from the excited level was collected by a photomultiplier and plotted as a function of the frequency of one of the lasers. The frequency of the second laser is held fixed at a given detuning from resonance. The experimental apparatus is similar to that which we have described in two previous papers. A longer detailed manuscript, including experimental details, is being prepared for publication.

The results of an experiment in which the fixed-frequency laser is tuned to exact resonance with the $F=$ 1 to $F=2$ transition and the second laser is tuned through the two-photon resonance are shown in Fig. (2a). The fluorescence is proportional to the population in the excited level, $\sigma_{22}$. In Fig. (2b) the theoretical prediction, Eq. (9), is shown for comparison with the experimental results. At the relatively low intensities used here the hole is quite narrow; in fact, the full width at half maximum (FWHM) is $9 \mathrm{MHz}$.

From Eq. (9) we can show that this curve is well approximated by the difference between two Lorentzians. The FWHM of the Lorentzian hole, in the low-field limit $\alpha, \beta \ll \Gamma_{a}, \Gamma_{b}$, is

$$
\mathrm{FWHM}=\left(\alpha^{2}+\beta^{2}\right) / 2\left(\Gamma_{a}+\Gamma_{b}\right) .
$$

The finite resolution of the experimental apparatus smears the hole somewhat, but the depletion is still almost complete at resonance. This is a quite pronounced effect even though we do not have nearly an ideal three-level system. There are actually eight Zeeman sublevels in the ground state and five in the excited state coupled by various transition dipole moments in our system.

Figure 3 is a composite drawing showing the effect of tuning the fixed frequency laser off resonance. There is still a sharp minimum at the double resonance, but the lineshape is asymmetric. More importantly, the maximum population in level 2 is obtained when the lasers are both somewhat detuned from resonance. The maximum actually occurs in the second trace when $\delta_{a}$ $=-12 \mathrm{MHz}$. The exact optimum depends on the lifetimes and Rabi frequencies of the particular transitions. If the intensities of the two lasers are increased, the width of the hole increases and one must tune even

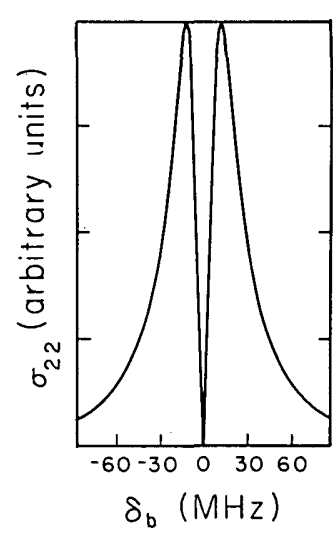

(a)

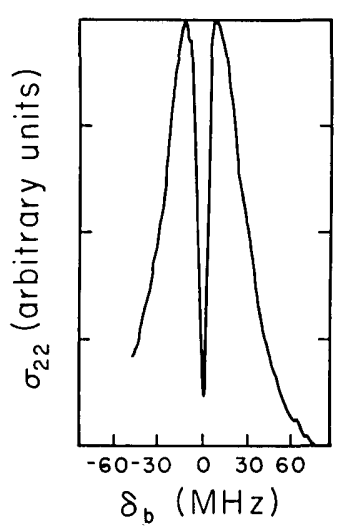

(b)
Fig. 2. (a) Experimentally observed excited-state population $\sigma_{22}$. The fixed-frequency laser is at exact resonance, $\delta_{a}=0$, with an intensity of $23 \mathrm{~mW} / \mathrm{cm}^{2}$. The second laser is detuned $\delta_{b}$ from exact resonance, with an intensity of $54 \mathrm{~mW} / \mathrm{cm}^{2}$. (b) Theoretical prediction of excited-state population $\sigma_{22}$. The fixed-frequency laser is at exact resonance, $\delta_{a}=0$. The second laser is detuned $\delta_{b}$ from exact resonance.

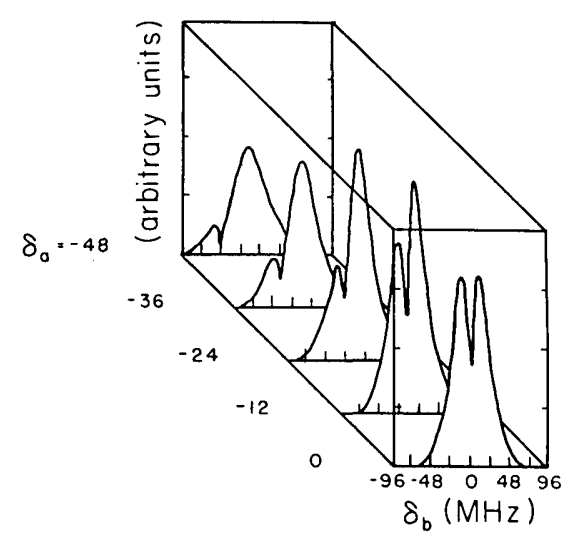

Fig. 3. Off-resonance spectra. The experimentally observed excited-state population for $\delta_{a}=0,-12,-24,-36$, and -48 $\mathrm{MHz}$ detuning from exact resonance. 
further from resonance to obtain appreciable excitation.

These effects have played a role in a number of previous experiments. They were directly observed in an optical pumping experiment by Alzetta et al. ${ }^{3}$ Often, however, the effect is not noticed because the bandwidth of the laser is too large or the lasers are tuned to maximize the ionization signal, fluorescence signal, etc., without regard to the exact detuning from resonance. The formalism developed here and in Refs. 1 and 2 refers only to a system with two ground-state levels. It is easily extended to more-general cases. There are simply more equations analogous to Eqs. (2a) and (2c) and somewhat more complex constants of the motion, but the results are exactly the same.

It is possible to circumvent this difficulty and get efficient excitation in at least two ways. One can use lasers with bandwidths larger than their Rabi frequencies so that the coherence is washed out, or one can detune the lasers from the two-photon resonance. It is clear, nonetheless, that this effect can have a large influence on the efficiency of laser extraction and should be taken into account in designing such experiments.

We also wish to point out that this technique of coherent trapping of populations forces the atom into a coherent superposition of ground states. This opens the interesting prospect of looking for ground-state quantum beats with this coherent superposition of the ground states.

This work was supported in part by the Office of Naval Research through contract N0014-68-A-0091 and by the National Science Foundation through grant SER77-06917. We would like to thank Bruce Shore for helpful discussions on the theory of this effect.

* Present address: Aerospace Corporation, P.O. Box 92957, Los Angeles, California 90009.

\section{References}

1. E. Arimondo and G. Orriols, "Nonabsorbing atomic coherences by coherent two-photon transitions in a threelevel optical pumping," Nuovo Cimento Lett. 17, 333 (1976).

2. R. Whitley, "Double Optical Resonance," doctoral dissertation (University of Rochester, Rochester, N.Y., 1977); see also B. W. Shore, Lawrence Livermore Laboratory Report 76-292.

3. G. Alzetta, A. Gozzini, L. Moi, and G. Orriols, "An experimental method for the observation of r.f. transitions and laser beat resonances in oriented $\mathrm{Na}$ vapour," Nuovo Cimento 36B, 5 (1976). 